\title{
Strategies for Pulling the Goalie in Hockey
}

\author{
David Beaudoin and Tim B. Swartz *
}

\begin{abstract}
This paper develops a simulator for matches in the National Hockey League (NHL) with the intent of assessing strategies for pulling the goaltender. Aspects of the approach that are novel include breaking the game down into finer and more realistic situations, introducing the effect of penalties and including the home-ice advantage. Parameter estimates used in the simulator are obtained through the analysis of an extensive data set using constrained Bayesian estimation via Markov chain methods. Some surprising strategies are obtained which do not appear to be used by NHL coaches.
\end{abstract}

Keywords : Bayes constrained estimation, Markov chain Monte Carlo, National Hockey League, Simulation.

*David Beaudoin is Assistant Professor, Département Opérations et Systèmes de Décision, Faculté des Sciences de l'Administration, Pavillon Palasis-Prince, Bureau 2636, Université Laval, Québec (Québec), Canada G1V0A6. Tim Swartz is Professor, Department of Statistics and Actuarial Science, Simon Fraser University, 8888 University Drive, Burnaby BC, Canada V5A1S6. Both authors have been partially supported by research grants from the Natural Sciences and Engineering Research Council of Canada. Beaudoin thanks the Mathematics and Statistics Department at Laval for the use of its computing resources. The authors are appreciative of helpful comments provided by the Editor, the Associate Editor and two referees. 


\section{INTRODUCTION}

We motivate our problem by considering game three of the semifinal series (tied at one game apiece) between the Quebec Remparts and the Shawinigan Cataractes in the QMJHL (Quebec Major Junior Hockey League) held on April 21st, 2009. The home team, Shawinigan, is leading 3-0 in the third period, much to the delight of the capacity crowd at the Bionest Centre. However, the referees call two consecutive penalties to the Cataractes with 13:06 and 12:22 minutes remaining. With his team about to play 5-on-3, the Remparts' famous head coach, Patrick Roy, elects to "pull" his goalie in order to go 6-on-3 (i.e. replace his goaltender with a skater). Perhaps the best goaltender to ever play the game, Roy was known as a fighter. This bold move shows he is no different in his coaching duties. He believes that the Remparts have to score during the two-man advantage to have a reasonable shot at coming back in the game, so he decides to go all-in. The move backfires as the Cataractes score an empty-net goal with 11:58 left in the third period. The game ends 4-1 in favor of Shawinigan. Some angry fans called the strategy "stupid" in postgame radio shows. Others thought it was a good decision, even though it did not turn out favorably in this particular game, reminding everyone that this very same strategy led to a goal 16 days earlier in the Remparts previous series against Cape Breton. So who was right? Does this strategy improve a team's probability of winning the game? This is a question that would be best served via an objective statistical analysis.

Before going further and to add some context to the above paragraph, we provide some basic facts about the game of ice hockey, or hockey as it is known in North America. Hockey is played with six players per side consisting of five "skaters" and a goaltender. The goaltender generally remains close to his "net" and attempts to prevent "goals" which

occur when the "puck" enters the net. Typically, skaters are on the ice for intervals of less than one minute, and are continuously replaced due to the exhaustive fast-paced 
style of the game. During a game, "penalties" occur for player infractions and these are assessed by the on-ice officials (referees and linesmen). When a minor or a major penalty occurs (two minutes and five minutes in duration, respectively), the offending player is sent to the "penalty box" and his team is forced to play "shorthanded". This period of time is known as a "power-play" for the opponent and it provides them with a better opportunity to score a goal. If a goal is scored by the opponent during a power-play resulting from a minor penalty, the offending player is released from the penalty box. "Offsetting" penalties occur when each team is assessed a penalty of the same type; in the case of offsetting major penalties, the two players are sent to the penalty box but the teams do not play shorthanded. For multiple penalties that are not offsetting, the rules are more complex and we refer the reader to www.nhl.com/ice/page.htm?id=26299.

Hockey is played at the highest level in the National Hockey League (NHL) which consists of 30 teams located in the United States and Canada. A NHL season is 82 games in length where a game is 60 minutes long, divided into three "periods" of 20 minutes. At the end of regulation time in the NHL, the team which has the greatest number of goals wins the game. If a game is tied at the end of regulation time, the game is extended for five minutes of "sudden-death overtime" whereby the first team to score wins the match. In overtime, the two teams play shorthanded, 4-on-4 with respect to skaters. If the game remains tied at the end of overtime, there is a "shootout" where three players for each team take a "penalty shot". The team with the most penalty goals wins the game. If the match is still tied, a single penalty shot is taken by each team, and this continues in a sudden-death fashion until one team has scored and the other team has not scored. The team which wins the game is awarded two points in the standings. If a team loses in overtime or in a shootout, they are awarded a single point.

Finding better strategies for pulling the goalie in hockey is important to teams as it 
may provide them with a few more points in the standings every year. This can be the difference between making the playoffs or not. It can also result in home-ice advantage in a playoff series. In other words, using improved strategies can provide additional millions of dollars to a team. Yet, the topic is seldom discussed and very few statistical analyses have investigated the problem. Coaches simply rely on conventional wisdom, or on what has been done for decades in the world of hockey. According to St. Louis Blues head coach Andy Murray, "I think a guide rule is if you're down by two goals, you pull him with about two minutes remaining. Or if you're down by one goal, you're looking at the one-minute mark." But is that really the correct strategy? And what about more complex situations like the one described above, where a team trailing by three goals has a two-man power-play with 12 minutes left?

The first paper on the subject of pulling the goaltender was written by Morrison (1976). It contains a major flaw, as pointed out by Morrison and Wheat (1986): the analysis compares the strategy of pulling the goalie at time $t$ with the strategy of never pulling the goalie. In other words, this paper omits the case where a coach pulls his goalie later at some time $t_{1}>t$. Morrison and Wheat (1986) correct the mistake and investigate the optimal time for pulling the goalie when teams are of equal strength. The paper argues that teams have a general scoring rate of $L$ goals per minute. When a team pulls its goaltender, its scoring rate increases to $2.67 \mathrm{~L}$ goals per minute, and the opponent's scoring rate increases to $7.83 \mathrm{~L}$ goals per minute when facing the open net. This assumption is referred to as the proportional assumption. Erkut (1987) generalizes the method to the situation where teams have different scoring rates. Nydick and Weiss (1989) argue that the proportional assumption for estimating the scoring rates in situations where a team pulls its goalie may not be adequate. Therefore, they suggest the use of situational rates which are constant across teams. Their work shows that results can be quite different 
depending on the estimation method chosen.

Washburn (1991) proposes a dynamic programming approach for determining the optimal time to pull the goalie. The author mentions that previous work concerns the probability that the team currently trailing scores before the opponent scores, and also before time expires in the game. He raises an important point: "Strictly speaking, scoring first is neither necessary nor sufficient for victory." A team trailing by a goal might tie the game but give up another goal before regulation ends. Washburn (1991) finds the optimal decision with respect to a recursive equation.

More recently, Berry (2000) assumes that the time until a goal is scored follows an exponential distribution. Accordingly, he calculates the probability that a team trailing by one goal scores within the next $t$ minutes and scores before their opponent. The author estimates various scoring rates by considering lower and upper bounds, claiming that "The NHL does not keep track (or at least I couldn't find them) of goals scored for the team that pulled their goalie."

Finally, Zaman (2001) considers the problem from a Markov chain point of view. The author defines seven possible states for the Markov chain: Goal A, Shot A, Zone B, Neutral, Zone A, Shot B, Goal B. He estimates transition probabilities based on data, and he argues that symmetry allows one to reduce the number of parameters to be estimated. The methodology suggests pulling the goalie when trailing by one goal with five to eight minutes left, depending on the current location of the puck (defensive/neutral/offensive zone).

This paper extends the approach of Berry (2000) in a number of ways to enhance the realism of the problem. We develop a simulation program to simulate hockey games under specified strategies with respect to pulling the goaltender. Under large numbers of simulations, we are able to approximate expected results and therefore assess strategies. 
Our approach incorporates penalties in the simulation, a non-negligible aspect of hockey. We also consider the effect of the home-ice advantage, and the impact of overtime and shootouts, reflecting the current state of affairs in the NHL. Previous papers are based upon general scoring rates, whose estimation combines all possible situations (e.g. 5-on-5, 5-on-4, 4-on-5, etc. with respect to the number of skaters). We simulate games keeping the situations distinct and we develop a Bayesian approach based on Markov chain methods to obtain the scoring rates. In addition, we are able to modify scoring rates according to whether a team is average, above average or below average. As a check of model adequacy, the simulation model mimics actual NHL games extremely well. The simulation program is very flexible, and we imagine that our contribution will be useful as more and more teams adopt sports analytics.

Although it is tempting to discuss "optimal" strategies with respect to pulling the goaltender, we believe that the notion of optimality is somewhat misguided. For example, suppose that a team is interested in the best time to pull its goaltender when trailing by a goal and the opponent has a penalty. Suppose further that this situation presents itself with 9 minutes remaining in a hockey game. With 9 minutes left in the game on a powerplay and trailing by one goal, the decision that faces a coach is whether the goaltender should be pulled now. He cannot ask himself whether he should pull the goaltender with 6 minutes left in the game as the situation may change. Most likely, one of the teams will have scored or the penalty will have expired. In determining optimality, we note that there are an enormous (possibly infinite) number of strategies concerning pulling the goaltender as complete strategies are based on pre-planned rules for every conceivable situation involving the score, the time remaining, the number of skaters on the ice, etc. Therefore, the best one might do is create a list of plausible strategies and determine optimality from the set. 
In our enhanced analysis which considers game situations, teams are faced with answering a simple question - should they pull the goalie now under the given situation? What we can do is investigate the choice in comparison to standard strategies such as pulling one's goalie with one minute remaining when trailing given the current situation. Therefore the focus of the paper is not on optimal strategies, but rather, we investigate the effect of pulling the goalie under situations of interest. We can assess whether pulling the goalie under a given situation is a wise decision. Moreover, there are many situations that are tenable and are worthy of investigation.

In Section 2, we describe the data collection process, an enormously tedious task that is essential in obtaining a realistic simulator. The data is taken from the 2007-2008 season of the NHL. Hence, the results (being sensitive to scoring rates) are only directly applicable to the NHL. In the process of collecting the data, various observations were made. We present these in a series of Remarks in Section 2. Some of the Remarks are surprising, while others address folklore that has not been previously investigated via data. Remark \#2 which concerns a comparison of penalty rates between home and visiting teams may even be an officiating concern for the NHL. In Section 3, we provide a description of the simulaton scheme where various assumptions are supported by statistical theory. The realism of the simulator is dependent on the estimation of scoring rates and the Bayesian estimation procedure is discussed in Section 4. In Section 5, we provide some of our simulation results. Some of the proposed strategies are provocative, and to our knowledge, have never been attempted. Our simulator is extremely flexible, and we encourage General Managers to investigate specific strategies tailored to their own teams and opponents. We conclude with a short discussion in Section 6. 


\section{DATA ANALYSIS}

We use the notation $a$-on- $b$ to denote the game situation where there are $a$ skaters on the ice for the team of interest and $b$ skaters for the opponent. Following conventional practice in the NHL, we assume that two teams never have their goaltenders pulled simultaneously and we assume that a team never pulls its goaltender if it results in the team having fewer skaters than its opponent. This leads to $m=25$ game situations as listed in Table 1 . Note that each of the five underscored game situations in Table 1 can be broken into two subcases according to whether the team of interest has pulled its goaltender. The underscored game situations receive special attention in Section 4.

\begin{tabular}{|c|c|c|c|c|c|c|c|}
\hline \multicolumn{4}{|c|}{ Opponent Goaltender Present } & \multicolumn{4}{|c|}{ Opponent Goaltender Removed } \\
\hline $6-$ on- 5 & 6 -on-4 & $6-$ on-3 & $\underline{5-o n-5}$ & 5 -on- 6 & 5 -on-5 & 4 -on-6 & $4-$ on- 5 \\
\hline$\underline{5-o n-4}$ & $\underline{5-o n-3}$ & 4-on-5 & $\underline{4-o n-4}$ & 4-on-4 & 3 -on-6 & 3 -on-5 & 3 -on-4 \\
\hline 4-on-3 & 3-on-5 & 3-on-4 & 3-on-3 & & & & \\
\hline
\end{tabular}

Table 1: The $m=25$ game situations subdivided according to whether the opponent (second team) has removed its goaltender. Note that each of the five underscored game situations can be broken into two subcases according to whether the team of interest has pulled its goaltender.

The simulation study from Section 3 requires the scoring and penalty rates under each of the 25 game situations. To be more specific, we need the distributions of the times of the following five events under each game situation:

- a goal scored by the road team

- a goal scored by the home team 
- a two-minute penalty called on the road team

- a two-minute penalty called on the home team

- an offsetting minor penalty

This investigation omits 4-minute and 5-minute penalties because they are rare. Future work could easily incorporate these events. For parameter estimation as described in Section 4 , we require the following match data under each game situation:

- total time played in minutes

- number of road goals

- number of home goals

- number of two-minute penalties called on the road team

- number of two-minute penalties called on the home team

- number of offsetting minor penalties

Data are not readily available in the form listed above. For example, whereas the total number of penalties during a game is typically recorded, it is not the case that the penalties are summarized in tandem with the corresponding game situation. However, the information can be determined by looking carefully at detailed game records. This enables us to determine the starting and ending times of every penalty.

We have collected data on all games from the 2007-2008 NHL season. The National Hockey League's official website (www.nhl.com) provides detailed ice time for each player, including goaltenders. Our resultant data file contains over 28,000 rows where each row 
corresponds to one of the five events described above with its corresponding situation, or a game situation change (for example the expiration of a penalty, or a pulled goaltender).

The data enables us to compute the sample mean times (in minutes) of the five events for each team under each game situation. In Table 2, we present aggregate results for road teams under 8 of the $m=25$ game situations. Note that the sample mean times for home teams can be deduced from Table 2. We now provide a series of remarks based on the data. Remarks 3, 4 and 5 can be inferred directly from Table 2 . The remaining remarks are obtained from Table 2 combined with the 17 unreported situations (which comprise only $1.1 \%$ of the total minutes played during the 2007-2008 NHL season).

Remark \#1 As expected, home teams perform better than road teams. The number of goals scored is 3497 to 3182 in favor of home teams (2.9 versus 2.6 per game).

Remark \#2 Road teams are called for more penalties than home teams in an 11:10 ratio (5433 to 4939). This is in line with the common perception that referees are influenced by the home crowd.

Remark \#3 Combining road and home statistics, a goal is scored by either team every 13.7 minutes when playing 5-on-5 with both goaltenders. Common sense dictates that more goals ought to be scored when teams are playing 4-on- 4 with both goaltenders, which is the case here since a goal is scored every 12.1 minutes.

Remark \#4 For the pulled goalie strategy to be effective, a necessary condition is that the team pulling the goaltender has to score at a higher rate when playing 6-on-5 than 5-on-5 with both goaltenders. Combining road and home statistics, teams playing 6-on-5 score a goal every 8.5 minutes, which is way below the sample means of 28.6 and 26.2 minutes when playing 5 -on-5 with both goaltenders for the road 
and home teams, respectively. Therefore, the decision to pull a goalie when trailing late in the game seems promising.

Remark \#5 One feature of this work that has not previously been investigated is the option of pulling the goalie during a power-play (just like the example described in Section 1). Based on the data, teams that decide to put an extra attacker on the ice to create a 6-on-4 situation score a goal every 5.5 minutes and allow an empty-net goal every 4.8 minutes. In other words, not only does the strategy force a goal to be scored more quickly, but teams that pull their goalie are almost as likely to score a goal as to allow one.

Remark \#6 Here is one very important argument in favor of pulling the goalie that has not been discussed in the past: sending an extra attacker on the ice seems to induce more penalties called on the team that is trying to defend its lead. In the 2007-2008 NHL season, 652.4 minutes were played with a goalie pulled. During that time, 44 penalties were called on the team which pulled the goalie versus 84 penalties on their opponents. This is almost a 1:2 ratio. From a slightly different perspective, penalties are called on the opponent more frequently when playing 6-on-5 (every 7.4 minutes and every 7.9 minutes for the road and home teams respectively) versus playing 5-on-5 with both goaltenders (every 12.2 minutes and every 13.3 minutes for the road and home teams respectively). As a result, pulling your goalie not only increases the scoring rates, but it also makes your team much more likely to get a power-play! 


\begin{tabular}{|c|r|r|r|r|r|r|}
\hline Game & Total & \multicolumn{5}{|c|}{ Sample Mean Times in Minutes } \\
Situation & Time in & Road & Home & Road Minor & Home Minor & Offsetting \\
(Road-on-Home) & Minutes & Goal & Goal & Penalty & Penalty & Minors \\
\hline 5-on-5 & 54841.0 & 28.6 & 26.2 & 12.2 & 13.3 & 135.8 \\
4-on-4 & 2306.6 & 27.8 & 21.4 & 17.3 & 12.3 & 329.5 \\
5-on-4 & 7733.4 & 9.8 & 70.3 & 21.5 & 27.0 & 2577.8 \\
4-on-5 & 8390.9 & 78.4 & 9.5 & 22.2 & 24.2 & 8390.9 \\
\hline 6-on-5 & 237.9 & 8.5 & 3.1 & 18.3 & 7.9 & 119.0 \\
6-on-4 & 55.9 & 5.1 & 5.6 & 18.6 & 7.0 & - \\
\hline 5-on-6 & 272.0 & 2.6 & 8.5 & 7.4 & 11.3 & 136.0 \\
4-on-6 & 53.8 & 4.1 & 6.0 & 13.5 & 53.8 & 53.8 \\
\hline
\end{tabular}

Table 2: Statistics corresponding to road teams (2007-2008 NHL season) for 8 of the $m=25$ game situations. The first four rows refer to the most common game situations where neither goaltender has been pulled, the fifth and six rows refer to the most common game situations where the road goaltender has been pulled and the last two rows refer to the most common game situations where the home goaltender has been pulled.

\section{SIMULATION MODEL}

At any time during a simulated game, we are concerned with seven possible events that can occur:

- the road team scores a goal

- the home team scores a goal

- the road team gets called for a two-minute penalty 
- the home team gets called for a two-minute penalty

- the referee calls offsetting minors

- if at least one player from either team is in the penalty box, a penalty expires

- a team pulls its goalie

Let $X_{1}, X_{2}, X_{3}, X_{4}$ and $X_{5}$ be the times in minutes until the first five events described above occur, respectively. We assume that the five random variables follow the Exponential distribution. Berry (2000) uses the Exponential assumption regarding the time between goals and he mentions that several other authors have relied on this hypothesis (Anderson-Cook and Thornton 1998; Berry, Reese and Larkey 1999; Danehy and Lock 1995). Recall that if the number of occurrences of a given event in $t$ minutes is Poisson $(\lambda t)$, then the time in minutes until the first event is Exponential $(\lambda)$ where $1 / \lambda$ is the mean of the Exponential distribution. The Poisson distribution can be motivated by thinking of goals occurring as Bernoulli trials over a large number of possessions.

A game begins with teams playing 5-on-5. We simulate the following random variables which all correspond to the 5-on-5 situation:

$X_{1} \sim \operatorname{Exp}\left(\lambda_{1,5-\text { on-5 }}\right)$ where $\lambda_{1,5-\text { on-5 }}$ is the Poisson parameter for a road goal

$X_{2} \sim \operatorname{Exp}\left(\lambda_{2,5-\text { on-5 }}\right)$ where $\lambda_{2,5-\text { on-5 }}$ is the Poisson parameter for a home goal

$X_{3} \sim \operatorname{Exp}\left(\lambda_{3,5-\text { on-5 }}\right)$ where $\lambda_{3,5-\text { on-5 }}$ is the Poisson parameter for a road penalty

$X_{4} \sim \operatorname{Exp}\left(\lambda_{4,5-\mathrm{on}-5}\right) \quad$ where $\lambda_{4,5-\mathrm{on}-5}$ is the Poisson parameter for a home penalty

$X_{5} \sim \operatorname{Exp}\left(\lambda_{5,5-\mathrm{on}-5}\right) \quad$ where $\lambda_{5,5-\mathrm{on}-5}$ is the Poisson parameter for offsetting minors

The $\lambda$ parameters are estimated as described in Section 4 . The event $i \in(1, \ldots, 5)$ that occurs next is the one whose variable $X_{i}$ is the smallest. If a goal is scored, the same process is repeated. If a penalty is called, the game situation changes and we now 
simulate according to the parameters associated with the new game situation. Simulating under a game situation that involves a minor penalty, if all five random variables take values which are larger than 2.0, the penalty expires and the teams go back to playing 5-on-5.

Now, how is a goalie pulled in a simulated game? We have defined several indicators that dictate the coach's strategy with respect to pulling the goalie. More specifically, one needs to input the time that the goalie is pulled when trailing by $g$ goals under each game situation $s$ for all values of $g=1, \ldots, 5$. For example, one may want to pull the goalie when currently playing 5-on-5 with 57 minutes played (i.e. three minutes left in the third period) if trailing by a single goal. As a result, when a team trails by one goal with three minutes or less left in the game, the simulator pulls the goalie whereby the Exponential parameters reset to the 6-on-5 situation, and the five random variables are simulated accordingly.

The simulator is therefore very flexible as it allows the user to try any strategy involving pulling the goalie. It is also possible to start all simulated games at time $t$ under any current game situation with either team trailing by $g$ goals. The output is the average number of points

$$
\mathrm{ANP}=\left(2 n_{2}+1 n_{1}+0 n_{0}\right) / M
$$

for the team of interest based on the simulation of $M=n_{2}+n_{1}+n_{0}$ games where

- $n_{2}=$ number of wins

- $n_{1}=$ number of losses in overtimes or shootouts

- $n_{0}=$ number of losses in regulation time

Determining the winning team in a shootout is handled via the Bernoulli distribution with the Bernoulli parameter $p=0.5$. We do not think there is a strong rationale 
for giving either the home or the road team an advantage once the overtime period is over. The crowd and the referees do not have much impact during shootouts. The data substantiates the claim as there is no statistically significant difference in shootout victory rates when comparing home and road teams. Although there is mild evidence that some teams may be superior at shootouts to other teams, we have not incorporated this effect into our simulator.

\section{BAYESIAN PARAMETER ESTIMATION}

As described in Section 3, the NHL game simulator requires distributional parameters for the generation of Exponential variates corresponding to the times of goals and penalties. A simple approach to parameter estimation involves the calculation of sample rates corresponding to the events of interest, and this is done in the case of penalties. However, in the case of goal scoring, the simple approach fails to take into account constraints which are imposed by logic but may not be satisfied when using sample rates. For example, it is clear that a team should score at a higher rate when playing 5 -on-3 than when playing 5-on-4. Sample rates can sometimes be out of alignment due to the rarity of the situation (e.g. 3-on-3). We take a Bayesian approach to parameter estimation for goal scoring where constraints are handled in a convenient fashion via a sampling framework. The Bayesian approach also allows the inclusion of prior beliefs.

Consider then the statistical model

$X_{i s}^{h}=$ total goals scored by the $i$ th home team in situation $s \sim \operatorname{Poisson}\left(n_{i s}^{h} \theta_{i s}\right)$

$X_{i s}^{r}=$ total goals scored by the $i$ th road team in situation $s \sim \operatorname{Poisson}\left(n_{i s}^{r} f \theta_{i s}\right)$

where $i=1, \ldots, N$ and there are $N=30$ NHL teams. Since the random variables in (2) are based on goals scored by the team of interest (and not goals against), we can reduce 
the number of game situations from $m=25$ to $\tilde{m}=20$ where we note that the scoring rates are assumed equal for the pairs of underscored game situations listed in Table 1. In the statistical model $(2), n_{i s}^{h}$ is the total number of minutes played by the $i$ th team when at home in game situation $s$ and $n_{i s}^{r}$ is the total number of minutes played by the $i$ th team when on the road in game situation $s$. The fraction $f$ is introduced so that the ratio of the goal scoring rate for home versus away is constant across all situations. The unknown parameters $\theta_{i s}$ are team and situation specific.

Our model assumes that individual team scoring rates arise from a population of league wide scoring rates

$$
\theta_{i s} \sim \operatorname{Gamma}\left(a_{s}, b_{s}\right)
$$

where the parameters $a_{s}$ and $b_{s}$ have independent prior distributions

$$
a_{s} \sim \operatorname{Gamma}\left(\alpha_{a s}, \beta_{a s}\right) \text { and } b_{s} \sim \operatorname{Gamma}\left(\alpha_{b s}, \beta_{b s}\right)
$$

The hyperparameters $\alpha_{a s}, \beta_{a s}, \alpha_{b s}$ and $\beta_{b s}, s=1, \ldots, \tilde{m}$ are set in an empirical Bayes fashion by considering the sample scoring rates. The Gamma hyperparameters are chosen such that $\alpha_{a s}>1$ and $\alpha_{b s}>1$. We impose a Uniform $(0,1)$ prior for $f$ in $(2)$ according to the widely held belief that home-ice confers an advantage. The primary parameter of interest in our analysis is

$$
\lambda_{s}=a_{s} b_{s}
$$

which denotes the league wide scoring rate under situation $s=1, \ldots, \tilde{m}$. In Table 3 , we present the logical constraints imposed on the $\lambda_{s}$ parameters for the 12 situations involving an opponent with a goaltender. A separate set of constraints is available for the 8 situations where the opponent does not have a goaltender. The notation in Table 3 is changed such that the subscript $s$ is written in a more accessible way (i.e. 5-on-4, 
6-on-5, etc.). Table 3 is presented such that parameters are constrained from above by parameters lying to the left or above the parameter of interest. Similarly, parameters are constrained from below by parameters lying to the right or below the parameter of interest. For example, Table 3 imposes the constraint $\max \left(\lambda_{6-\mathrm{on}-5}, \lambda_{4-\mathrm{on}-4}\right) \leq \lambda_{5-\mathrm{on}-4} \leq$ $\min \left(\lambda_{6-\mathrm{on}-4}, \lambda_{4-\mathrm{on}-3}\right)$.

\begin{tabular}{|l|l|l|l|}
\hline$\lambda_{6-\mathrm{on}-3}$ & & & \\
\hline$\lambda_{5-\mathrm{on}-3}$ & $\lambda_{6-\mathrm{on}-4}$ & & \\
\hline$\lambda_{4-\mathrm{on}-3}$ & $\lambda_{5-\mathrm{on}-4}$ & $\lambda_{6-\mathrm{on}-5}$ & \\
\hline$\lambda_{3-\mathrm{on}-3}$ & $\lambda_{4-\mathrm{on}-4}$ & $\lambda_{5-\mathrm{on}-5}$ & \\
\hline & & $\lambda_{4-\mathrm{on}-5}$ & $\lambda_{3-\mathrm{on}-4}$ \\
\hline & & & $\lambda_{3-\mathrm{on}-5}$ \\
\hline
\end{tabular}

Table 3: Constraints for the 12 situations where the opponent has a goaltender.

The posterior distribution arising from the Bayesian model is complex, constrained and high-dimensional. Consequently, the posterior means of the $\lambda$ 's cannot be obtained analytically. Fortunately, this is a problem that is well-suited to a sampling framework using Markov chain Monte Carlo methods (see Gilks, Richardson and Spiegelhalter, 1996). We iteratively simulate from the full conditional distributions, repeating a simulation step whenever a generated parameter $\lambda_{s}$ does not satisfy its constraint. The full conditional distributions for $f$ and $\theta_{i s}$ are convenient for variate generation and are given by

$$
\begin{gathered}
{[f \mid \cdot] \sim \operatorname{Gamma}\left(1+\sum_{i} \sum_{s} X_{i s}^{r}, 1 / \sum_{i} \sum_{s} n_{i s}^{r} \theta_{i s}\right)} \\
{\left[\theta_{i s} \mid \cdot\right] \sim \operatorname{Gamma}\left(X_{i s}^{h}+X_{i s}^{r}+a_{s}, b_{s} /\left(1+b_{s} n_{i s}^{h}+b_{s} n_{i s}^{r} f\right)\right)}
\end{gathered}
$$

where $i=1, \ldots, N$ and $s=1, \ldots, \tilde{m}$. 
The full conditional distributions for $a_{s}$ and $b_{s}$ are non-standard, and we introduce Metropolis-within-Gibbs steps to complete the Markov chain algorithm. Specifically, we generate $\mu \sim \operatorname{Uniform}(0,1)$ and generate $a_{s}$ according to its constrained prior distribution which also serves as the proposal density. We denote the previously generated value of $a_{s}$ as $a_{s *}$. We then set $a_{s}=a_{s *}$ if

$$
\mu>\exp \left(\left(a_{s}-a_{s *}\right) \sum_{i} \log \left(\theta_{i s}\right)-N \log \Gamma\left(a_{s}\right)+N \log \Gamma\left(a_{s *}\right)-N\left(a_{s}-a_{s *}\right) \log \left(b_{s}\right)\right) .
$$

For $b_{s}$, we similarly generate $\mu \sim \operatorname{Uniform}(0,1)$ and generate $b_{s}$ according to its constrained prior distribution which also serves as the proposal density. We denote the previously generated value of $b_{s}$ as $b_{s *}$. We then set $b_{s}=b_{s *}$ if

$$
\mu>\exp \left(-\sum_{i} \theta_{i s} / b_{s}+\sum_{i} \theta_{i s} / b_{s *}-N a_{s} \log \left(b_{s}\right)+N a_{s} \log \left(b_{s *}\right)\right) .
$$

The Markov chain algorithm described above has been coded in the $\mathrm{R}$ programming language. We obtain the posterior means and posterior standard deviations of the $\lambda$ 's. We note that the standard deviations provide us with the opportunity to consider parameters that deviate from the league wide rates. For example, we can add/subtract one standard deviation from the posterior means to obtain team scoring rates that are above/below the league wide rates. Although proprietary constraints prevent us from releasing the $\lambda$ estimates, the posterior standard deviations of the $\lambda$ 's tend to be larger for situations with larger posterior means. The posterior standard deviations are also affected by the amount of data (i.e. number of minutes) corresponding to the game situations.

The Markov chain algorithm provides the posterior mean 0.95 for the fraction $f$ used to delineate the home ice advantage. In the NHL game simulator, if one is interested in the road team, then the posterior means of the $\lambda$ 's are simply scaled by $f=0.95$. 


\section{SIMULATION RESULTS}

Our simulator is flexible as it can generate matches from any time point and game situation under any set of proposed strategies. The simulator also appears to mimic actual NHL games extremely well. For example, the average number of goals per game by the road and home teams during the 2007-2008 NHL season are 2.65 and 2.91 respectively. This compares favorably with the simulated average number of goals per game, 2.68 and 2.91, by the road and home teams respectively.

We investigate several strategies under different scenarios. The effectiveness of each strategy is measured by a team's average number of points ANP given by (1) based on $M=150$ million simulated games. Let $y_{i}$ be the number of points obtained in the $i$ th simulated hockey game and note that $y_{i}$ takes on the values 0,1 and 2 . Then the half length of the approximate $95 \%$ confidence interval for the mean of ANP is $1.96 s / \sqrt{M}<$ $1.96 / \sqrt{M}$ since $\max \left(s^{2}\right)=\sum \max \left(y_{i}-\bar{y}\right)^{2} /(M-1) \leq \sum 1^{2} /(M-1)=M /(M-1) \approx 1$. Therefore choosing $M=150$ million simulated games provides estimates that are typically precise to three decimal places.

An analysis of the time when goaltenders were pulled by their coaches during the 2007-2008 NHL season shows that this move is typically done with 1 minute remaining if a team is trailing by one goal and with 1:30 remaining in the case of a two-goal deficit. The strategy is generally adopted by NHL coaches no matter the game situation, except for shorthanded situations, in which case the goaltender is almost never pulled. Let us call the above decision rules the current strategy. In Tables 4 through 7 , we investigate four scenarios along with various strategies which are listed in order of effectiveness as measured by their ANP. In each scenario, the opponent uses the same strategy as the team of interest. The four scenarios are given as follows: 
A - The road team is trailing by 1 goal with 3 minutes left. Both teams are playing at full strength (5-on-5).

B - The home team is trailing by 2 goals with 6 minutes left. Both teams are playing at full strength (5-on-5).

C - The home team is trailing by 1 goal with 1:54 minutes left. The home team is playing shorthanded (4-on-5) as they just got called for a penalty.

D - The scenario described in Section 1 where the road team is trailing by 3 goals with 12:22 left. The road team has a 5-on-3 power-play with 2:00 minutes and 1:16 minutes remaining in the penalties.

The best strategy with respect to scenario A (see Table 4) is to be extremely aggressive by pulling the goalie until either the game ends, or until the road team ties the game. In other words, the road team should go all-in. Note that ANP decreases if you slightly modify this strategy by leaving your goalie in net when playing shorthanded. The difference between strategies 3 and 4 is that the road team does not wait until there is one minute left in the game to pull its goalie if trailing by a single goal in any power-play situation. In such a case, the road coach pulls the goalie immediately.

In scenario B (see Table 5), the current strategy can be improved upon in various ways. Leaving the home goalie in net, the score will likely remain the same until there is 1:30 left in the game (this is the moment where NHL coaches start thinking about making a move). At this point, it is pretty much a lost cause for the home team. It's too late to reasonably hope for a comeback. The home team needs to be a lot more aggressive. They need to score quickly, even if it means increasing the risk of being scored against. Note that the all-in strategy does not do quite as well as the strategy which suggests pulling the goalie under any circumstance unless shorthanded. In other words, the roles 


\begin{tabular}{|c|l|c|}
\hline Strategy & \multicolumn{1}{|c|}{ Description } & ANP \\
\hline 1 & Pull goalie until the score is tied & 0.2527 \\
\hline 2 & Pull goalie until the score is tied unless shorthanded & 0.2512 \\
\hline 3 & $\begin{array}{l}\text { Current strategy except goalie also pulled in power-play } \\
\text { situations }\end{array}$ & 0.2116 \\
\hline 4 & Current strategy & 0.2045 \\
\hline
\end{tabular}

Table 4: The road team is trailing by 1 goal with 3 minutes left. Both teams are playing at full strength (5-on-5).

are reversed compared to scenario A where a coach was better off pulling the goalie even in shorthanded situations. This is in line with the common perception that if the home team gets a penalty with 5:30 left in the game, for instance, it should hold off pulling its goalie. If they can make it through the next two minutes without allowing a goal, there will still be 3:30 remaining in the game. The subtlety in strategy 1 relies on the fact that if the home team manages to cut the lead to a single goal fairly quickly (say, 5 minutes left), they should put their goalie back in net if playing 5-on-5. Once the game reaches the 57-minute mark (i.e. 3 minutes remaining) with teams at full strength, the home goalie should get pulled again if they are still trailing by one goal. The decision to pull the home goalie a second time with 3:00 left could probably be improved upon even more.

With respect to scenario $\mathrm{C}$ (see Table 6), the largest value for ANP occurs for the all-in strategy, where the home coach pulls the goalie until his team ties the score, or until the game is over. This shows that with as little time remaining as 1:54, a team trailing by one goal should be desperate and extremely aggressive in order to maximize their chances of getting at least one point in the game. The two strategies that performed best are the ones that involve pulling the goalie even in shorthanded situations (which is the case 


\begin{tabular}{|c|l|c|}
\hline Strategy & \multicolumn{1}{|c|}{ Description } & ANP \\
\hline 1 & $\begin{array}{l}\text { Pull goalie until the score is tied unless (a) shorthanded } \\
\text { or (b) trailing by 1 goal playing 5-on-5 in which case the } \\
\text { goalie is pulled if there are less than 3 minutes left }\end{array}$ & 0.0798 \\
\hline 2 & Pull goalie until the score is tied unless shorthanded & 0.0780 \\
\hline 3 & Pull goalie until the score is tied & 0.0771 \\
\hline 4 & $\begin{array}{l}\text { Current strategy except goalie also pulled in power-play } \\
\text { situations }\end{array}$ & 0.0583 \\
\hline 5 & Current strategy & 0.0512 \\
\hline
\end{tabular}

Table 5: The home team is trailing by 2 goals with 6 minutes left. Both teams are playing at full strength (5-on-5).

when each simulated game starts with 1:54 remaining).

With respect to scenario D (see Table 7), if we assume that Patrick Roy's intention was to pull his goalie not only during the 5-on-3 situation, but also for the 5-on-4 ensuing power-play, then his game plan corresponds to strategy 2. Indeed, it seems logical that if a coach decides to pull his goalie in power-play situations when trailing by 3 goals with 12 minutes left, then he is willing to do so with any lesser amount of time left. That is exactly what the simulation scheme does: every time Quebec gets a power-play in simulated games, they pull their goalie. From the results presented in the Table 7, it looks like Roy's move was a good one. It did increase the expected number of points compared to three of the listed strategies. However, it is important to note that Roy's strategy would have been a good one in an NHL game. Scoring rates are higher in the QMJHL, which suggests waiting a little longer before pulling a goalie (compared to the NHL). Therefore, we can only conclude that pulling the goalie with 12:22 left was a good 


\begin{tabular}{|c|l|c|}
\hline Strategy & \multicolumn{1}{|c|}{ Description } & ANP \\
\hline 1 & Pull goalie until the score is tied & 0.0761 \\
\hline 2 & $\begin{array}{l}\text { Current strategy except goalie also pulled in } \\
\text { shorthanded situations }\end{array}$ & 0.0614 \\
\hline 3 & Current strategy & 0.0409 \\
\hline 4 & Never pull the goalie & 0.0351 \\
\hline
\end{tabular}

Table 6: The home team is trailing by 1 goal with 1:54 minutes left. The home team is playing shorthanded (4-on-5) as they just got called for a penalty.

decision in an NHL context. This claim cannot be supported in the QMJHL context until we obtain scoring and penalty rates from this league. Further, from the infinite collection of possible strategies, we did find one (strategy 1) that beats Patrick Roy's strategy.

In summary, the simulations suggest that NHL coaches are too conservative. The current strategy is easily outperformed in terms of ANP with more aggressive decisions regarding pulling the goaltender. All of the papers mentioned in Section 1 similarly conclude that goalies should be pulled earlier. An important question concerns the benefit that a team realizes over the course of a full season of 82 games by using more aggressive strategies. We simulate 4 million games between average road and home teams under three general strategies. The objective is to compare the ANP using the current strategy with the ANP using more aggressive strategies. The results are given in Table 8 and are listed in increasing order of aggressiveness.

From Table 8, an average team can increase its expected number of points by 1 over the course of an 82-game season by simply pulling the goalie when trailing by any number of goals with less than 3 minutes left. A more aggressive approach results in an improvement over the current strategy by 1.5 points. Without providing all of the details, the more 


\begin{tabular}{|c|l|c|}
\hline Strategy & \multicolumn{1}{|c|}{ Description } & ANP \\
\hline 1 & $\begin{array}{l}\text { Pull goalie until the score is tied in any power-play or } \\
\text { 4-on-4 situation. If playing 5-on-5, pull goalie with 3 } \\
\text { minutes left if trailing by 1 goal and with 6 minutes left } \\
\text { if trailing by 2 goals. Never pull the goalie shorthanded }\end{array}$ & 0.0914 \\
\hline 2 & $\begin{array}{l}\text { Current strategy except goalie also pulled in power-play } \\
\text { situations }\end{array}$ & 0.0813 \\
\hline 3 & Pull goalie until the score is tied unless shorthanded & 0.0752 \\
\hline 4 & $\begin{array}{l}\text { Current strategy except goalie also pulled in 5-on-3 } \\
\text { situations }\end{array}$ & 0.0671 \\
\hline 5 & Current strategy & 0.0661 \\
\hline
\end{tabular}

Table 7: The scenario described in Section 1 where the road team is trailing by 3 goals with 12:22 left. The road team has a 5-on-3 power-play with 2:00 and 1:16 minutes remaining in the penalties.

aggressive approach involves pulling the goaltender when shorthanded, even-strength and on power-plays with increasing time remaining and various goal deficits. Finding even better strategies is an obvious research question of interest. The gain in terms of expected number of points might turn out to be 2-3 points per season. While that may not seem to be a major improvement at first glance, note that the seeding of 13 teams (43\% of all teams) would have been higher than their actual seeding had they obtained an extra 2.1 points during the 2007-2008 season. 


\begin{tabular}{|l|c|c|}
\hline \multicolumn{1}{|c|}{ Strategy } & ANP per game & ANP per season \\
\hline Current strategy & 1.0208 & 83.7 \\
\hline $\begin{array}{l}\text { Pull goalie when trailing as soon as } \\
\text { there is less than 3 minutes left }\end{array}$ & 1.0330 & 84.7 \\
\hline An even more aggressive approach & 1.0385 & 85.2 \\
\hline
\end{tabular}

Table 8: Comparison of general strategies over the course of an 82-game season.

\section{CONCLUDING REMARKS}

This paper investigates strategies involving pulling the goaltender. The approach is the most comprehensive to date as it takes into account penalties, home-ice advantage and breaks games down into finer situations. A constrained empirical Bayes model is used to facilitate parameter estimation.

The results are surprising and suggest innovative strategies for teams to improve. Over the course of a season, the implementation of improved strategies by a team may result in meaningful differences such as a higher seeding for the playoffs. One general result is that teams that are trailing should pull their goaltenders much earlier when awarded with a power-play than when playing 5-on-5. We realize that pulling the goaltender at much earlier times is a difficult decision for coaches. Coaches face intense pressure from the media and fans, and they are typically questioned on results even if strategies are sensible. Coaches have acted conservatively for decades and they obviously require the support of General Managers in order to implement provocative strategies.

Of course, if every team were to adopt improved strategies for pulling the goaltender, an advantage would cease to exist. This is the evolutionary process of sport; an innovation is introduced, success is observed, and the innovation is copied. Upon full adoption of the 
innovation, an advantage is no longer conferred. As an example of this, see Lewis (2006) who chronicles the rise in importance of the left tackle position in the National Football League.

An important aspect of the paper is that the results may be tailored for specific pairs of teams by using team-specific parameters. Also, although our attention has been focused on strategies for pulling the goaltender, it is clear that our general purpose NHL simulator has applications to various problems involving prediction. For example, teams may want to know the impact of substituting a particular combination of players with an alternative combination of players in a specific game situation (e.g. power-plays). Such an application requires parameters specific to various player combinations.

\section{REFERENCES}

Anderson-Cook, C.M. and Thornton, T. (1998). "Measuring hockey's special teams efficiency", Chance, 11(4), 26-34.

Berry, S.M. (2000). "My triple crown - First leg: pulling the goalie", Chance, 13(3), 56-57.

Berry, S.M., Reese, C.S. and Larkey, P.L. (1999). "Bridging different eras in sports", Journal of the American Statistical Association, 94, 661-686.

Danehy, J.T. and Lock, R.H. (1995). "CHODR - Using statistics for predict college hockey", Stats, 13, 10-14.

Erkut, E. (1987). "Note: More on Morrison and Wheat's 'Pulling the goalie revisited"', Interfaces, 17, 121-123.

Gilks, W.R., Richardson, S. and Spiegelhalter, D.J. (editors) (1996). Markov Chain Monte Carlo in Practice, London: Chapman and Hall. 
Lewis, M.M. (2006). The Blind Side, New York: W.W. Norton and Company.

Morrison, D.G. (1976). "On the optimal time to pull the goalie: A Poisson model applied to a common strategy used in ice hockey", TIMS Studies in Management Sciences, Volume 4, North Holland, New York, 137-144.

Morrison, D.G. and Wheat, R.D. (1986). "Misapplications reviews: Pulling the goalie revisited", Interfaces, 16, 28-34.

Nydick, R.L. and Weiss, H.J. (1989). "More on Erkut's 'More on Morrison and Wheat's 'Pulling the goalie revisited"', Interfaces, 19, 45-48.

Washburn, A. (1991). "Still more on pulling the goalie", Interfaces, 21, 59-64.

Zaman, Z. (2001). "Coach Markov Pulls Goalie Poisson", Chance, 14(2), 31-35. 